\title{
0 papel da telemetria no diagnóstico da Fibrilhação Auricular no Acidente Vascular Cerebral criptogénico - A experiência de uma Unidade de AVC.
}

\author{
The role of telemetry in the diagnosis of \\ atrial fibrillation in criptogenic Strokes - a Stroke Unit experience.
}

\author{
António Ferreira, Cátia Barreiros, Duarte Silva,Lúcia Meireles-Brandão, Edgar Torre, Carmélia Rodrigues \\ Unidade Local de Saúde do Alto Minho - Hospital de Santa Luzia, Estrada de Santa Luzia, Viana do Castelo, Portugal
}

\begin{abstract}
Resumo
A embolia cardíaca por Fibrilhação Auricular (FA) paroxística não diagnosticada é responsável por uma parte substancial dos Acidentes Vasculares Cerebrais (AVC) isquémicos classificados como criptogénicos. As atuais orientações recomendam a monitorização electrocardiográfica nas primeiras 24-48h após a admissão hospitalar. Os autores pretenderam avaliar o número de FA, de novo, identificadas por telemetria em doentes com Acidente Isquémico Transitório (AIT)/AVC isquémico, sem outra etiologia identificada, numa Unidade de AVC, e sua caracterização.
\end{abstract}

Material e Métodos: Foram incluídos os doentes admitidos numa Unidade de AVC tipo C, entre Maio a Dezembro de 2015, e seleccionados aqueles com diagnóstico de AIT/AVC sem etiologia identificada e sem FA conhecida ou previamente documentada, que tiveram registo electrocardiográfico por telemetria durante um período mínimo de 24 horas. A equipa médica foi responsável pela revisão da telemetria para identificação de FA. A análise dos dados foi efectuada com o programa SPSS v23.

Resultados: Dos 217 doentes admitidos foram seleccionados 79. A idade média foi de 73,9 anos e distribuição por sexo masculino/feminino de $53,2 \%$ e $46,8 \%$, respectivamente. 70 doentes apresentaram AVC isquémico (88,6\%) e 9 doentes AIT (11,4\%). Dos 79 doentes, 12 (15,2\%) apresentaram ritmo de FA no registo por telemetria (FA paroxística: 11; FA persistente: 1) e destes, em 11 (91\%) doentes foi alterada estratégia terapêutica com inicio de hipocoagulação.

Discussão: 0 número de FA detectadas na presente série foi sobreponível aos dados aferidos noutros estudos. A telemetria constitui um método importante no diagnóstico de FA em doentes com AVC isquémico que, de outro modo, poderiam ser definidos como criptogénicos. 0 registo electrocardiográfico por telemetria permite assim assumir mais precocemente uma correcta estratégia terapêutica.

Palabras clave: Telemetria. Fibrilación auricular. AVC criptogénico

\section{Introdução}

A Fibrilhação Auricular (FA) é um factor de risco independente e a causa de pelo menos $20 \%$ de todos os Acidentes Vasculares Cerebrais (AVCs) isquémicos ${ }^{1}$. Os doentes com FA têm um risco 5 vezes superior de AVC comparativamente a doentes sem esta arritmia². Apresentam ainda maior mortalidade e são frequentemente mais incapacitantes do que AVCs secundários a outras causas ${ }^{3}$. A FA pode ocorrer de modo paroxístico ou persistente, mas o risco de embolia cerebral parece ser semelhante em ambas ${ }^{4}$.

Um acidente vascular cerebral (AVC) isquémico denomina-se por criptogénico quando não é identificada uma etiologia

\begin{abstract}
Undetected cardiac embolism by Atrial Fibrillation (AF) is responsible for a substantial number of ischemic strokes classified as cryptogenic. Current guidelines recommend electrocardiographic monitoring for the first 2448 hours after hospital admission. The authors intended to evaluate the number of new onset AF identified by telemetry in patients with transient ischemic attack (TIA) / ischemic stroke, without other etiology identified, in a stroke unit, and its characterization.

Material and Methods: Patients admitted in a type $\mathrm{C}$ unite stroke from May to December 2015 were included in the study and selected those diagnosed with TIA / stroke without identified etiology, without know AF or documented on admission of the current hospitalization, and who had electrocardiographic register by telemetry for a minimum period of 24 hours. The medical team was responsible for reviewing telemetry data with the purpose of AF identification. Data analysis was performed with SPSS v23.

Results: Of 217 patients admitted in our stroke unit, 79 were selected. The mean age was 73.9 years and $53,2 \%$ were male. 70 patients had an ischemic stroke (88.6\%) and 9 patients had a TIA (11.4\%). Of the 79 patients, in $12(15.2 \%)$ was identified AF rhythm in the telemetry data (paroxysmal AF: 11; persistent AF: 1) and of these, 11 (91\%) patients had their therapeutic strategy altered through initiation of anticoagulation therapy. Discussion: The number of AF detected in this series was similar to the data from other studies. Telemetry is an important exam in the diagnosis of $\mathrm{AF}$ in patients with ischemic stroke, that otherwise, could be defined as cryptogenic. The electrocardiographic recording by telemetry permits an earlier and correct therapeutic approach.
\end{abstract}

Keywords: Telemetry. Atrial fibrillation. Cryptogenic stroke.

após realização de estudo etiológico exaustivo. A embolia cardiogénica por FA paroxística, não detectada, é provavelmente responsável por uma parte substancial deste tipo de AVC.

0 tratamento correcto após um Acidente Isquémico Transitório (AIT) ou AVC implica a identificação de uma FA se esta estiver presente. A terapêutica antitrombótica nos doentes em que não é conhecida FA consiste na antiagregação com acido acetilsalicílico ou clopidogrel ${ }^{5}$. No entanto, se houver diagnóstico de FA deve ser iniciada hipocoagulação pois, nestes casos, a terapêutica antiagregante plaquetária é inferior à terapêutica anticoagulante na prevenção secundária de $\mathrm{AVC}^{6}$, sendo que, comparativamente à antiagregação pla- 
quetária, a varfarina, por exemplo, reduz em $40 \%$ o risco de AVCs recorrentes? ${ }^{7}$. Sendo assim, a não identificação de FA resulta num risco desnecessariamente aumentado de recorrência de AVC, pelo que o seu reconhecimento é de extrema importância.

As guidelines actuais recomendam um electrocardiograma de 12 derivações e pelo menos 24 horas de monitorização cardíaca após um AVC ou AIT.

0 diagnóstico de FA em doentes com AIT ou AVC pode ser difícil e a detecção tardia é comum. Um ECG de rotina de 12 derivações prontamente identifica uma FA persistente, no entanto a FA é assintomática e paroxística em mais de $50 \%$ dos casos $^{8}$. Ziegler et.al ${ }^{9}$ descrevem, num grupo de doentes nos quais foi detectada FA após AVC, a ocorrência desta arritmia em média 5 minutos por dia e a maioria não apresentava FA em $90 \%$ dos dias nos quais foram seguidos. Nos últimos anos diversos estudos têm avaliado várias técnicas de monitorização electrocardiográfica no internamento que ainda não são comtempladas nas actuais guidelines. A realização de ECGs seriados, a monitorização prolongada e repetitiva por Holter, a monitorização electrocardiográfica por telemetria (detecção manual e automática) e detectores de eventos implantáveis ${ }^{10-15}$ podem aumentar a detecção de FA após um evento cerebral isquémico. Apesar de existirem estudos prospectivos comparativos ainda não foi definida a melhor modalidade diagnóstica.

Em ambulatório, para já, a evidência disponível suporta a realização de monitorização cardíaca de doentes com AVC ou AIT seleccionados, nomeadamente: idade avançada, doença cardíaca subjacente, aumento aurícula esquerda, enfartes corticais sugestivos de etiologia embólica ou contracções auriculares prematuras frequentes durante a monitorização cardíaca em internamento.

Em suma, com novos métodos de diagnóstico, o número de doentes com AVC que apresentam FA parece ser maior do que 0 estimado previamente. Neste sentido, e de acordo com as orientações, é importante uma monitorização electrocardiográfica nas primeiras 24-48h após a admissão hospitalar, sendo que a melhor técnica para 0 fazer ainda não está definida.

A Unidade Local de Saúde do Alto Minho é constituída por duas Unidades Hospitalares (Hospital Santa Luzia em Viana do Castelo e Hospital Conde Bertiandos em Ponte de Lima) que abrangem uma população estimada de 244836 habitantes e dispõe de um total de 408 camas de internamento. 0 Hospital de Santa Luzia em Viana do Castelo é um hospital distrital e abrange uma área maioritária do distrito de Viana do Castelo. Na Unidade de AVC (UAVC) deste hospital a monitorização electrocardiográfica, através de telemetria sem software de detecção automática, é apenas possível desde Junho/2015. Apesar de demonstradas outras técnicas de deteção da FA como superiores a esta monitorização, este é o meio disponível no nosso hospital, sendo a gestão dos recursos e adaptação a cada realidade médica hospitalar determinante na escolha.
Neste sentido, o presente trabalho teve como objectivo principal avaliar o número de $\mathrm{FA}$, de novo, diagnosticadas por telemetria na UAVC e determinar o número de doentes em que a identificação desta FA por telemetria alterou o plano terapêutico.

\section{Material e métodos}

População: Foi realizado um estudo observacional, descritivo e retrospectivo, que decorreu no período de Junho a Dezembro de 2015 no qual foram incluídos todos os doentes admitidos na Unidade de AVC do Hospital de Santa Luzia. Foram seleccionados os doentes com: idade superior a 18 anos; diagnóstico de AIT/AVC sem etiologia identificada e sem FA documentada previamente ou na admissão do actual internamento; registo electrocardiográfico por telemetria durante um período mínimo de 24 horas, até 48 horas. Foram excluídos todos os doentes com: idade inferior a 18 anos, AVC hemorrágico; AVC isquémico com etiologia identificada (FA, vasculites, oclusão de grandes vasos, enfarte agudo do miocárdio, trombo no ventrículo esquerdo, forâmen ovale patente, hipercoagulabilidade,...); telemetria com duração inferior a 24 horas; pacemaker na câmara auricular e diagnóstico de AVC não confirmado.

Metodologia: Os traçados electrocardiográficos por telemetria foram registados no aparelho com marca Mindray-iMEC10®, com início de monitorização na admissão do doente na UAVC. A revisão dos traçados foi realizada pela equipa médica de acordo com a indicação de sintomatologia pelos doentes ou por alarmes estabelecidos no software de monitorização, nomeadamente: assistolia, arritmia, taquicardia/fibrilação ventricular, couplets ou triplets, frequência cardíaca inferior a 60 ou superior a 100. A equipa de enfermagem foi sensibilizada para a detecção destes alarmes e subsequente reconhecimento de ritmo de FA.

Foi considerada FA paroxística todas as alterações electrocardiográficas compatíveis com FA e duração de pelo menos 30 segundos intercaladas com ritmo sinusal. 0 diagnóstico de AIT e AVC foi baseado na clínica e diferenciado em segundo tempo por método de imagem (TAC ou RM) pela presença ou ausência de lesão isquémica. A investigação diagnóstica de AVC decorreu de acordo com as guidelines da European Stroke Organization ${ }^{16}$

A colheita de informação demográfica, dados clínicos e informação definidora de escalas foi realizada através da revisão de processo clínico.

Análise estatística: Os dados obtidos foram analisados através do programa SPSS $®$ versão 23. A análise dos dados demográficos foi realizada por análise descritiva de dados e apresentada através da média e desvio padrão. Para comparação de variáveis entre grupos foram aplicados testes não paramétricos com um $\alpha=0,05$.

\section{Resultados}

Foram admitidos na UAVC no período de Junho a Dezembro de 2015 um total de 217 doentes. 79 doentes foram seleccionados com base nos critérios de inclusão estabelecidos e 
os restantes foram excluídos de acordo com os critérios de exclusão indicados previamente, nomeadamente: 40 doentes com AVCs hemorrágicos ou diagnóstico não confirmado de AVC; 27 doentes com identificação de outra etiologia que não FA; 35 apresentaram FA previamente; 15 apresentaram FA de novo no momento da admissão no serviço de urgência; 21 não tiveram registo de telemetria ou 0 tempo de duração da mesma foi inferior a 24 horas (Figura 1).

Dos 79 doentes seleccionados, $70(88,6 \%)$ corresponderam a AVCs e os restantes $9(11,4 \%)$ a AlTs.

A idade média foi de 73,9 anos, $42 \%$ eram do sexo masculino e $87,3 \%$ tinham autonomia completa para as actividades da vida diária. $25,3 \%$ dos doentes tiveram AVC isquémico no passado e $88,6 \%$ tinham factores de risco cardiovascular (FRCV) conhecidos previamente, nomeadamente, 61 $(77,2 \%)$ com hipertensão arterial, $36(45,6 \%)$ com dislipidemia e 24 (30,4\%) diabetes mellitus tipo 2. 0 valor médio da pontuação na escala NIHSS à admissão foi de 5,5 (desvio padrão de $\pm 4,9$, valor mínimo de 0 e máximo de 21). Na admissão hospitalar 6 doentes foram submetidos a trombólise, dos quais apenas um teve identificação de FA no registo por telemetria. $54 \%$ dos doentes apresentaram aumento do volume da aurícula esquerda. Relativamente à terapêutica de ambulatório prévia, 14 doentes estavam sob antiagregação plaquetária e 2 doentes encontravam-se hipocoagulados com varfarina. Os dados demográficos e caracterização dos doentes, classificação Oxfordshire Community Stroke Project (OCSP) e a distribuição pela escala de Rankin modificada na data da alta encontram-se descritos na Tabla 1.

Legenda: n -número; FRCV - Factores de Risco Cardiovasculares; NIHSS - National Institutes of Health Stroke Scale; DP - Desvio Padrão; OCSP - Oxfordshire Community Stroke Project Classification; TACI - total anterior circulation infarcts, $\mathrm{PACl}$ - partial anterior circulation infarcts, $\mathrm{LACl}$ - lacunar circulation infarcts, $\mathrm{POCl}$ - posterior circulation infarcts; mRankin: Escala de Rankin modificada.

Tabla 1. Caracterização da amostra e evento isquémico.

\begin{tabular}{|c|c|}
\hline Parâmetro & Valor \\
\hline População $-\mathrm{n}$ & 79 \\
\hline Género M/F - $\mathrm{n}(\%)$ & $42 / 37(53,3 \% / 46,8 \%)$ \\
\hline Idade (anos) - média (DP) & $73,97(11,63)$ \\
\hline Grau de Dependência - $\mathrm{n}(\%)$ & \\
\hline Total & $1(1,3 \%)$ \\
\hline Parcial & $9(11,4 \%)$ \\
\hline Autónomo & $69(87,3 \%)$ \\
\hline FRCV - n (\%) & $70(88,6 \%)$ \\
\hline Sim & $9(11,4 \%)$ \\
\hline Não & \\
\hline Classificação evento isquémico & $5,5 \pm 4,9$ \\
\hline NIHSS admissão - média (DP) & \\
\hline Classificação OCSP $-\mathrm{n}$ (\%) & $34(48,6 \%)$ \\
\hline LACl & \\
\hline
\end{tabular}

\begin{tabular}{|c|c|}
\hline Parâmetro & Valor \\
\hline $\mathrm{PACl}$ & $18(25,1 \%)$ \\
\hline $\mathrm{TACl}$ & $7(10 \%)$ \\
\hline $\mathrm{POCl}$ & $10(14,3 \%)$ \\
\hline $\mathrm{TACl}$ e POCl & $1(1,4 \%)$ \\
\hline mRankin & \\
\hline 0 & $27(34,2 \%)$ \\
\hline 1 & $13(16,5 \%)$ \\
\hline 2 & $15(19 \%)$ \\
\hline 3 & $11(13,9 \%)$ \\
\hline 4 & $9(11,4 \%)$ \\
\hline 5 & $4(5,1 \%)$ \\
\hline
\end{tabular}

Em 12 doentes (15,2\%) foi identificada FA no registo por telemetria, das quais, 9 em doentes com AVC e 3 em doentes com AIT. 11 doentes apresentaram FA paroxística e 1 doente apresentou FA sustentada após inicio de monitorização, ausente em ECGs prévios e no ECG da admissão do SU. Na figura 2 apresenta-se de forma esquemática a distribuição do evento isquémico e de FA na amostra estudada.

Neste grupo de doentes, apenas um doente tinha tido AVC isquémico no passado, 2 encontravam-se sob terapêutica antiagregante plaquetária e nenhum estava hipocoagulado. Todos os doentes tiveram um score CHA2DS2VASc igual ou superior a 1, e 4 doentes apresentaram um score HASBLED igual ou superior a 2. Dos 12 doentes com FA identificada no registo de telemetria foi iniciada hipocogulação em 11, excluindo-se um doente por apresentar score HASBLED 3 e quedas frequentes com traumas craneoencefálicos importantes.

Foi comparado o grupo de doentes em que foi identificada FA com o grupo de doentes sem identificação de FA (criptogénicos) relativamente aos FRCV prévios, classificação OCSP e pontuação na escala NIHSS à entrada e à saída não havendo evidência de diferenças estatisticamente significativas $(p>0,05)$.

\section{Discussão}

Os meios diagnósticos pelos quais é possível identificar uma FA são vários. Contudo, perante os meios disponíveis no nosso Hospital, em que a utilização de registo por Holter de 24 horas não é possível em regime de internamento, neste estudo foi utilizada a técnica de monitorização electrocardiográfica por telemetria sem software de detecção automática de FA.

A taxa de detecção de FA com monitorização entre 24 a 74 horas pode variar de 0 a 16\% com utilização de registos de Holter ou técnicas de monitorização contínua em internamento sem detecção automática ${ }^{17 .}$ A detecção de FA por telemetria neste estudo foi de $15,2 \%$, correspondendo ao descrito em alguns estudos. Através de meios de diagnóstico que permitem a monitorização em ambulatório por longos períodos, como a telemetria de ambulatório e outros dispositivos externos ou implantados, estão descritas taxas de detecção de FA até 28\%18,19, contudo 0 acesso a estes meios de diagnóstico ainda não está generalizado. 
Fig. 1. Processo de sele'çao da amostra. Legenda: UAVC - Unidade de Acidente Vascular Cerebral; AVC Acidente Vascular Cereblar; FA Fibrilhaçao auricular. N - Número.

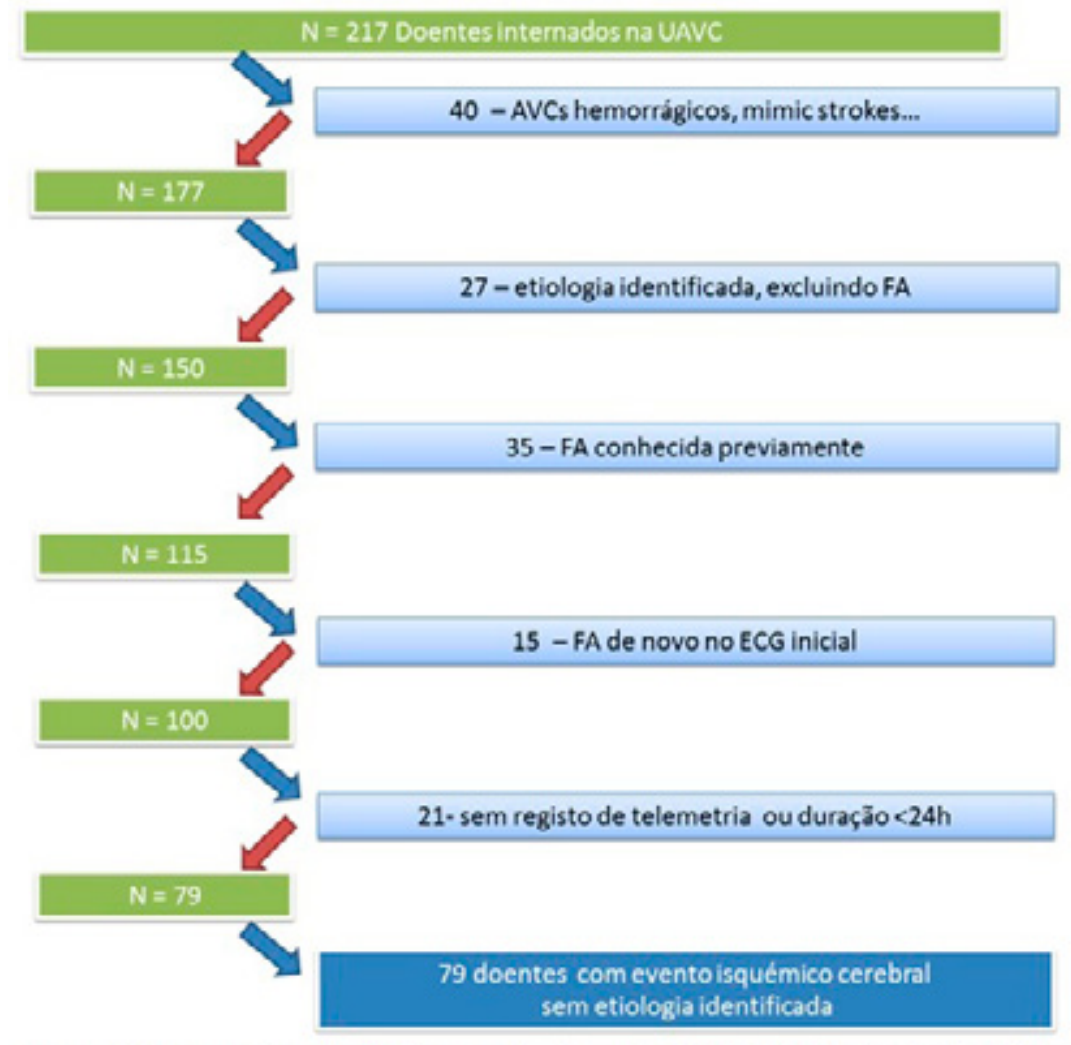

Fig. 2. Distribuçao dos eventos isquémicos cerebrais e prevaléncia de FA e respectivos subtipos. Legenda: AVC - Acidente Vascular Cerebral; AIT - Acidente Isquémico Transitorio; FA - Fibrilhaçao auricular. N - Número.

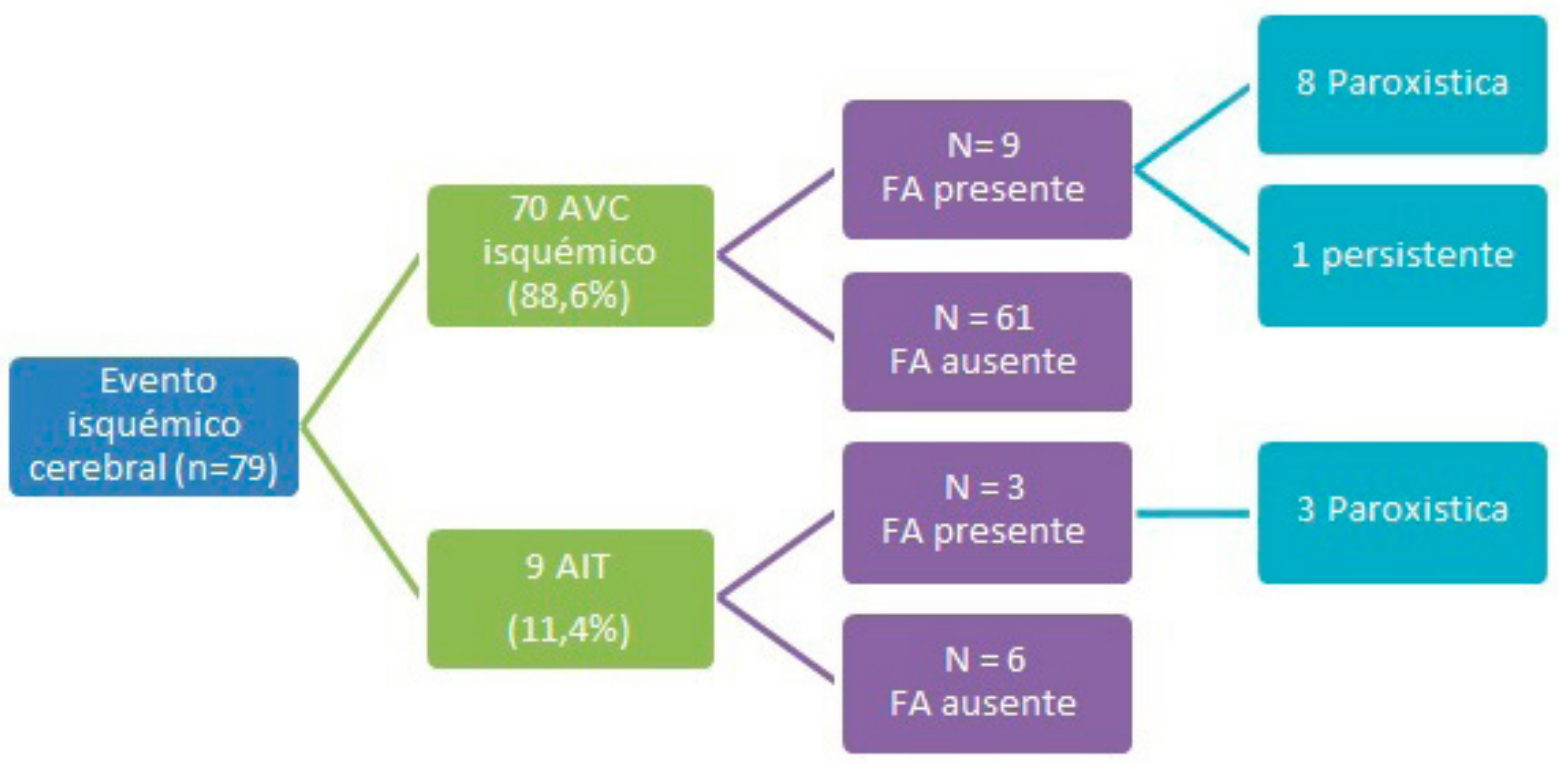


A técnica de monitorização contínua com detecção automática está descrita como superior aos registos que utilizam apenas a detecção manual ${ }^{10}$. No entanto, apesar do sistema de telemetria utilizado neste estudo não ser dotado de software de detecção automática, a identificação de FA na nossa amostra foi superior aos dados de algumas séries ${ }^{20}$. Realça-se, portanto, a importância do treino e do rigor sistemático na revisão dos traçados permitindo assim obter maiores taxas de diagnóstico de FA.

No presente estudo a duração da monitorização foi apenas de 48h. Atendendo a que o prolongamento do tempo de monitorização electrocardiográfica por telemetria pode aumentar a capacidade de aferição diagnóstica de $F A^{17}$, no futuro, dentro das limitações de recursos humanos e técnicos, na nossa unidade será aumentado o tempo de monitorização e realizada nova análises dos dados. A aplicação de outros recursos diagnósticos mais dispendiosos a uma população de doentes seleccionada poderá ser a estratégia de eleição para optimização da detecção de FA, associadamente às outras técnicas já disponíveis.

Na nossa amostra não foi identificado um subgrupo ou patologia em que a identificação de FA por telemetria fosse mais provável. Apesar de, como referido previamente, em ambulatório serem sugeridos alguns parâmetros de selecção para monitorização dos doentes, em regime de internamento ainda não estão identificados subgrupos que beneficiem de uma determinada técnica ou tempo de monitorização, encontrando-se em curso alguns estudos que poderão dar respostas mais concretas quer a nível de internamento quer de ambulatório.

0 início de hipocoagulação em 11 dos 12 casos em que foi detectada FA, constituiu uma importante alteração na prevenção secundária de AVC e prognóstico da doença. Consideramos uma estratégia de monitorização a manter e optimizar na nossa UAVC através do reforço positivo a toda a equipa de enfermagem e médica na melhoria da detecção de FA nos registos de telemetria.

\section{Conclusão}

0 número de FA detectadas na presente série foi semelhante aos dados aferidos noutros estudos. A telemetria constitui um método importante no diagnóstico de FA em doentes com AVCs isquémicos que, de outro modo, poderiam ser definidos como criptogénicos. 0 registo electrocardiográfico por telemetria permite assim assumir mais precocemente uma correcta estratégia terapêutica, e melhorar o prognóstico e a prevenção secundária de eventos.

\section{Bibliografia}

1. Tsang TS, Petty GW, Barnes ME, O'Fallon WM, Bailey KR, Wiebers DO, et al. The prevalence of atrial fibrillation in incident stroke cases and matched population controls in Rochester, Minnesota: changes over three decades. J Am Coll Cardiol 2003; 42: 93-100.

2. Wolf $P A$, Abbott $R D$, Kannel WB. Atrial fibrillation as an independent risk factor for stroke: the Framingham Study. Stroke 1991; 22: 983-88.

3. Lamassa M, Di Carlo A, Pracucci G, Basile AM, Trefoloni G, Vanni P, et al. Characteristics, outcome, and care of stroke associated with atrial fibrillation in Europe: data from a multicenter multinational hospital-based registry (the European Community Stroke Project). Stroke. 2001;32: 392-398.

4. Hohnloser SH, Pajitnev D, Pogue J, Healey JS, Pfeffer MA, Yusuf S, et al. Incidence of stroke in paroxysmal versus sustained atrial fibrillation in patients taking oral anticoagulation or combined antiplatelet therapy: an ACTIVE W Substudy. J Am Coll Cardiol. 2007;50:2156-2161.

5. Jauch EC, Saver JL, Adams HP Jr, Bruno A, Connors JJ, Demaerschalk BM, et al. Guidelines for the early management of patients with acute ischemic stroke: a guideline for healthcare professionals from the American Heart Association/American Stroke Association. Stroke 2013;44:870-947.

6. Camm AJ, Lip GY, De Caterina R, Savelieva I, Atar D, Hohnloser SH, et al. 2012 Focused update of the ESC Guidelines for the management of atrial fibrillation: an update of the 2010 ESC Guidelines for the management of atrial fibrillation - developed with the special contribution of the European Heart Rhythm Association. European Heart Journal 2012; 33: 2719-47.

7. Atrial Fibrillation investigators; Risk factors for stroke and efficacy of antithrombotic therapy in atrial fibrillation. Analysis of pooled data from five randomized controlled trials; Arch Intern Med. 1994 Jul 11;154(13):1449-57.

8. Lin HJ, Wolf PA, Benjamin EJ, Belanger AJ, D'Agostino RB, et al; Newly diagnosed atrial fibrillation and acute stroke. The Framingham Study. Stroke. 1995 Sep;26(9):1527-30.

9. Ziegler PD, Glotzer TV, Daoud EG, Wyse DG, Singer DE, Ezekowitz MD, et al. Incidence of newly detected atrial arrhythmias via implantable devices in patients with a history of thromboembolic events. Stroke. 2010;41:256-260.

10. Rizos T, Güntner J, Jenetzky E, Marquardt L, Reichardt C, Rüdiger Becker R, et al. Continuous stroke unit electrocardiographic monitoring versus 24-hour Holter electrocardiography for detection of paroxysmal atrial fibrillation after stroke. Stroke 2012;43:2689-94.

11. Douen AG, Pageau N, Medic S. Serial electrocardiographic assessments significantly improve detection of atrial fibrillation 2.6-fold in patients with acute stroke. Stroke 2008;39:480-2

12. Manina G, Agnelli G, Becattini C, Zingarini G, Paciaroni M. 96 Hours ECG monitoring for patients with ischemic cryptogenic stroke or transient ischaemic attack. Intern Emerg Med 2014;9:65-7.

13. Jabaudon D, Sztaizel J, Sievert K, Landis T, Sztajzel R. Usefulness of ambulatory 7-day ECG monitoring for the detection of atrial fibrillation and flutter after acute stroke and transient ischemic attack. Stroke 2004;35:1647-51.

14. Bhatt A, Majid A, Razak A, Kassab M, Hussain S, Safdar A. Predictors of occult paroxysmal atrial fibrillation in cryptogenic strokes detected by long-term non invasive cardiac monitoring. Stroke Res Treat 2011;2011:172074.

15. Cotter PE, Martin PJ, Ring L, Warburton EA, Belham M, Pugh PJ. Incidence of atrial fibrillation detected by implantable loop recorders in unexplained stroke. Neurology 2013;80:1546-50.

16. European Stroke Organisation (ESO) Executive Committee, ESO Writing Committee. Guidelines for management of ischaemic stroke and transient ischaemic attack 2008. Cerebrovasc Dis. 2008;25:457-507.

17. Grond M., Jauss M, Hamann G, Stark E, Veltkamp R, Nabavi D, et al; Improved Detection of Silent Atrial Fibrillation Using 72-Hour Holter ECG in Patients With Ischemic Stroke A Prospective Multicenter Cohort Study. Stroke, 2013; 44:3357-64.

18. Ziegler PD, Glotzer TV, Daoud EG, Wyse DG, Singer DE, Ezekowitz MD, et al. Incidence of newly detected atrial arrhythmias via implantable devices in patients with a history of thromboembolic events. Stroke. 2010;41:256-260.

19. Tayal AH, Tian M, Kelly KM, Jones SC, Wright DG, Singh D, et al. Atrial fibrillation detected by mobile cardiac outpatient telemetry in cryptogenic TIA or stroke. Neurology. 2008;71:1696-1701.

20. Sposato LA, Cipriano LE, Saposnik G, Ruíz Vargas E, Riccio PM, Hachinski V; Diagnosis of atrial fibrillation after stroke and transient ischaemic attack: a systematic review and meta-analysis, Lancet Neurol 2015; 14: 377-87. 